IVAN HLaváČEK (Praha)

JÁN Lovíš́EK (Bratislava)

\title{
CONTROL IN OBSTACLE-PSEUDOPLATE PROBLEMS WITH FRICTION ON THE BOUNDARY. OPTIMAL DESIGN AND PROBLEMS WITH UNCERTAIN DATA
}

Abstract. Four optimal design problems and a weight minimization problem are considered for elastic plates with small bending rigidity, resting on a unilateral elastic foundation, with inner rigid obstacles and a friction condition on a part of the boundary. The state problem is represented by a variational inequality and the design variables influence both the coefficients and the set of admissible state functions. If some input data are allowed to be uncertain a new method of reliable solutions is employed. We prove the existence of a solution to the above-mentioned problems on the basis of a general theorem on the control of variational inequalities.

Introduction. We consider some optimal design problems for an elastic pseudoplate (a plate with small bending rigidity). The bending of the pseudoplate is described by means of a shear model: the plate is deformed only by shear forces (see e.g. [1]). In classical elasticity theory a pseudoplate is a plate offering resistance to bending when only a shear is acting. We assume that a homogeneous and isotropic pseudoplate occupying a domain $\Omega \times(-H, H)$ of the space $\mathbb{R}^{3}$ is loaded by a transversal distributed force $p\left(x_{1}, x_{2}\right)$ perpendicular to the plane $O x_{1} x_{2}$. The pseudoplate rests on a unilateral elastic foundation and is supported unilaterally by a finite number of rigid obstacles (punchs). The role of design variables is played by (i) the thickness of the pseudoplate, (ii) the stiffness characteristic of the Winkler medium and (iii) the friction bound (slip limit). The design variables have to belong to a set of Lipschitz-continuous functions. The following cost func-

2000 Mathematics Subject Classification: 49J40, 74K20, 74P05, 93C30.

Key words and phrases: control of variational inequalities, optimal design, weight minimization, pseudoplate with obstacles, worst scenario, uncertain data. 
tionals are considered: (i) desired deflection, (ii) total reaction, (iii) compliance, (iv) intensity of shear forces and (v) weight of the pseudoplate. In the last case, i.e., the weight minimization problem, we introduce constraints, which express bounds for some mean values of the intensity of shear stresses.

The state problem is modelled by a variational inequality, where the control variables influence both the coefficients of the non-linear monotone operator and the set of admissible state functions. On the basis of a general existence theorem for a class of optimization problems with variational inequalities, we prove the existence of at least one solution to each of the optimal control problems mentioned above. The last one, i.e. the weight minimization, is treated via a penalty method.

In Section 4, we consider the same state problem with some uncertain data; namely, we assume that the distributed load, the coefficient of the elastic foundation and the slip limit are uncertain, being prescribed in some a priori given sets. Here we employ a method of reliable solutions (alias worst scenario method) (cf. [2], [3]). The existence of a "reliable" solution is proved on the basis of the general theorem introduced in Section 2.

In a forthcoming paper we shall propose approximate solutions of the problems mentioned above and present some convergence analysis.

1. Setting of the problem. Let the midplane of the pseudoplate occupy a given bounded domain $\Omega \subset \mathbb{R}^{2}$ with Lipschitz boundary $\partial \Omega$. We denote the standard Sobolev function spaces by $H^{k}(\Omega) \equiv W^{k, 2}(\Omega)$, $k=1,2$. Let the norm in $H^{k}(\Omega)$ be denoted by $\|\cdot\|_{k}$. In the following $L_{2}(\Omega)$ and $L_{\infty}(\Omega)$ denote the spaces of Lebesgue-square integrable and essentially bounded functions on $\Omega$, respectively, with the standard norms $\|\cdot\|_{0}$ and $\|\cdot\|_{\infty}$. The inner product in $L_{2}(\Omega)$ will be denoted by $\langle\cdot, \cdot\rangle_{0}$.

Let the boundary $\partial \Omega$ be decomposed as follows:

$$
\partial \Omega=\overline{\partial \Omega}_{D} \cup \overline{\partial \Omega}_{C}
$$

where $\partial \Omega_{D}$ and $\partial \Omega_{C}$ are open, non-empty and non-overlapping parts. On $\partial \Omega_{D}$ a homogeneous kinematic condition is prescribed, whereas on $\partial \Omega_{C}$ the pseudoplate is subject to a contact with friction.

The transversal displacements (deflections) $v$ belong to the space

$$
V=\left\{v \in H^{1}(\Omega): v=0 \text { on } \partial \Omega_{D}\right\} .
$$

Let $\tau_{13}, \tau_{23}$ denote the components of the stress field (shear stresses). We consider an isotropic and homogeneous elastic material. Assuming that the in-plane displacements vanish, we have the following stress-strain relations:

$$
\tau_{i 3}=K G \varepsilon_{i 3}=\frac{1}{2} K G \partial v / \partial x_{i}, \quad i=1,2,
$$


where $K$ is a shear correction factor (a positive constant) and $G=$ const is the elastic shear modulus.

For simplicity, we set $K G \equiv 1$.

Then the shear forces of the pseudoplate are

$$
V_{i 3}=\int_{-H}^{H} \tau_{i 3} d z=H \partial v / \partial x_{i}, \quad i=1,2 .
$$

The equilibrium equation of a pseudoplate without any internal obstacles or elastic supports has the form

$$
\partial V_{13} / \partial x_{1}+\partial V_{23} / \partial x_{2}+p=0
$$

or

$$
\operatorname{div}(H \operatorname{grad} v)=-p .
$$

On the boundary, we have

$$
V_{n 3}=\sum_{i=1}^{2} n_{i} V_{i 3}=H \partial v / \partial n,
$$

where $n$ denotes the unit outward normal. On the part $\partial \Omega_{C}$ we prescribe a slip limit $\mathcal{F}$ and the following friction conditions: either the surface force $\left|V_{n 3}\right|$ is less than the slip limit $\mathcal{F}$ and then the pseudoplate remains in its original position because of friction, or $\left|V_{n 3}\right|$ equals $\mathcal{F}$ and the pseudoplate can slip into a new equilibrium position in the opposite direction to the friction force. As a consequence, we have the following conditions on $\partial \Omega_{C}$ :

$$
\begin{aligned}
& H|\partial v / \partial n| \leq \mathcal{F}, \\
& H|\partial v / \partial n|<\mathcal{F} \Rightarrow v=0, \\
& H|\partial v / \partial n|=\mathcal{F} \Rightarrow \exists \lambda \geq 0 \text { such that } v=-\lambda V_{n 3} .
\end{aligned}
$$

Moreover, we consider several unilateral inner obstacles as follows. Given mutually disjoint subdomains $\Omega_{i}^{*}, i=1, \ldots, N$, such that $\overline{\Omega_{i}^{*}} \subset \Omega, \overline{\Omega_{i}^{*}} \cap$ $\overline{\Omega_{j}^{*}}=\emptyset$ for $i \neq j$ and constants

$$
O_{i} \geq H_{\max }, \quad i=1, \ldots, N,
$$

where $H_{\max }$ is a given upper bound for the half-thickness (see the definition of $U_{\mathrm{ad}}^{H}$ below), we consider the conditions

$$
v(x) \geq H(x)-O_{i} \quad \text { for a.a. } x \in \Omega_{i}^{*},
$$

for all $i=1, \ldots, N$.

On $\Omega \backslash \overline{\Omega^{*}}$, where $\Omega^{*}=\bigcup_{i=1}^{N} \Omega_{i}^{*}$, we consider a unilateral elastic foundation of Winkler type, i.e., the reaction force $\mathcal{R}_{w}$ is proportional to the negative part of the deflection $v$ :

$$
\mathcal{R}_{w}=\mathcal{Z}[v]^{-}, \quad[v]^{-}=\min \{v, 0\},
$$

where $\mathcal{Z}$ is a given non-negative coefficient (see the definition of $U_{\text {ad }}^{\mathcal{Z}}$ below). 
Let us use the virtual displacement principle to establish a variational formulation of the problem. To this end we introduce the set

$$
\mathcal{K}(H)=\{v \in V: v \text { satisfies }(1.2)\},
$$

a bilinear form

$$
a(H ; v, w)=\int_{\Omega} H \operatorname{grad} v \cdot \operatorname{grad} w d x
$$

and a form

$$
b(\mathcal{Z} ; v, w)=\int_{\Omega \backslash \Omega^{*}} \mathcal{Z}[v]^{-} w d x
$$

Here

$$
\begin{array}{r}
H \in U_{\text {ad }}^{H}=\left\{H \in C^{(0), 1}(\bar{\Omega})\right. \text { (i.e., Lipschitz-continuous functions) : } \\
\left.H_{\min } \leq H(x) \leq H_{\max },\left|\partial H / \partial x_{i}\right| \leq C_{i}^{H}, i=1,2\right\}
\end{array}
$$

with given positive constants $H_{\min }, H_{\max }, C_{i}^{H}$ such that $H_{\min }<H_{\max }$, $1 \in\left[H_{\min }, H_{\max }\right]$, and

$$
\mathcal{Z} \in U_{\text {ad }}^{\mathcal{Z}}=\left\{\mathcal{Z} \in C^{(0), 1}\left(\bar{\Omega} \backslash \Omega^{*}\right): 0 \leq \mathcal{Z}(x) \leq \mathcal{Z}_{\text {max }},\left|\partial \mathcal{Z} / \partial x_{i}\right| \leq C_{i}^{\mathcal{Z}}, i=1,2\right\}
$$

with given positive constants $\mathcal{Z}_{\max }, C_{i}^{\mathcal{Z}}$.

We define the friction functional $\Phi(H, \mathcal{F}): H^{1}(\Omega) \rightarrow \mathbb{R}$ by the formula

$$
\Phi(H, \mathcal{F})(v)=\int_{\partial \Omega_{C}} \mathcal{F}|v| d s+I_{\mathcal{K}(H)}(v)
$$

where

$$
\mathcal{F} \in U_{\text {ad }}^{\mathcal{F}}=\left\{\mathcal{F} \in C^{(0), 1}\left(\overline{\partial \Omega}_{C}\right): 0 \leq \mathcal{F}(s) \leq \mathcal{F}_{\text {max }},|d \mathcal{F} / d s| \leq C_{\mathcal{F}} \text { a.e. in } \partial \Omega_{C}\right\}
$$

with given positive constants $\mathcal{F}_{\max }, C_{\mathcal{F}}$, and $I_{\mathcal{K}(H)}(\cdot)$ is the indicatrix function of the set $\mathcal{K}(H)$. Finally, let a transversal load $p \in L_{2}(\Omega)$ and a constant specific weight $\omega$ be given.

In what follows, we set $e=\{H, \mathcal{Z}, \mathcal{F}\}$ and define

$$
U_{\mathrm{ad}}=U_{\mathrm{ad}}^{H} \times U_{\mathrm{ad}}^{\mathcal{Z}} \times U_{\mathrm{ad}}^{\mathcal{F}} \text {. }
$$

On the basis of the virtual displacement principle we introduce the following state problem: given any $e \in U_{\text {ad }}$, find $u(e) \in \mathcal{K}(H)$ such that

$$
\begin{aligned}
& a(H ; u(e), v-u(e))+b(\mathcal{Z} ; u(e), v-u(e)) \\
& +\Phi(H, \mathcal{F})(v)-\Phi(H, \mathcal{F})(u(e)) \geq\langle p-2 \omega H, v-u(e)\rangle_{0}
\end{aligned}
$$

for all $v \in \mathcal{K}(H)$.

Later on, we shall prove that the variational inequality (1.7) has a unique solution $u(e)$ for any $e \in U_{\text {ad }}$. 
Next, let us consider several optimal design problems. First, we introduce cost functionals. The simplest will be

$$
\mathcal{L}_{\mathrm{DD}}(v)=\int_{\Omega}(v-z)^{2} d x
$$

where $z \in L_{2}(\Omega)$ is a given function, or

$$
\mathcal{L}_{\text {ISS }}(v)=\int_{\Omega}|\operatorname{grad} v|^{2} d x
$$

which is closely related to the integral of the intensity of shear stresses, i.e.,

$$
\int_{\Omega} 3\left(\tau_{13}^{2}+\tau_{23}^{2}\right) d x=\frac{3}{4} \int_{\Omega}|\operatorname{grad} v|^{2} d x .
$$

The third cost functional will be the compliance

$$
\mathcal{L}_{\mathrm{COM}}(e, v)=\int_{\Omega}(p-2 \omega H) v d x .
$$

Let $\theta$ be any (fixed) function in $H_{0}^{1}(\Omega)$ such that $\theta=1$ on $\Omega^{*}$ a.e. and let $\mathcal{Z}^{0}$ be an extension of the function $\mathcal{Z} \in U_{\text {ad }}^{\mathcal{Z}}$ by zero on $\Omega^{*}$. We define

$$
\mathcal{L}_{\mathrm{TR}}(e, v)=\int_{\Omega}\left(H \operatorname{grad} v \cdot \operatorname{grad} \theta+\left(\mathcal{Z}^{0}[v]^{-}-p+2 \omega H\right) \theta\right) d x .
$$

This functional represents the resultant of transverse reactive forces on the inner obstacles. Let us justify the definition of $\mathcal{L}_{\mathrm{TR}}$ in detail: for any $v \in$ $\mathcal{K}(H) \cap H^{2}(\Omega)$ we decompose each subdomain $\Omega_{i}^{*}$ into the set

$$
S_{i}(v):=\left\{x \in \Omega_{i}^{*}: v(x)>H-O_{i}\right\}
$$

and its complement $Q_{i}(v)=\Omega_{i}^{*} \backslash S_{i}(v)$. Define

$$
Q(v)=\bigcup_{i=1}^{N} Q_{i}(v) .
$$

Obviously, $v=H-O_{i}$ on $Q_{i}(v)$. We introduce the following set:

$$
X(\Omega)=\left\{\theta \in H_{0}^{1}(\Omega): \theta=1 \text { a.e. on } \Omega^{*}\right\} .
$$

Lemma 1.1. If the solution $u(e)$ of the state problem (1.7) belongs to $H^{2}(\Omega)$, then

$$
\mathcal{L}_{\mathrm{TR}}(e, u(e))=\int_{Q(u(e))}(-p+2 \omega H-\operatorname{div}(H \operatorname{grad} u(e))) d x,
$$

i.e., it has the same value for all $\theta \in X(\Omega)$.

Proof. Let us show that

$$
N_{z}(e, u(e)) \equiv-\operatorname{div}(H \operatorname{grad} u(e))+\mathcal{Z}^{0}[u(e)]^{-}-p+2 \omega H=0
$$

in $\Omega \backslash Q(u(e))$ a.e. 
Consider a point $x^{0} \in S_{i}(u(e))$. There is a ball $B_{\varrho}\left(x^{0}\right) \subset S_{i}(u(e))$ and a non-negative function $\varphi \in C_{0}^{\infty}\left(B_{\varrho}\left(x^{0}\right)\right)$ such that $\varphi>0$ on a closed ball $B_{\varrho / 2}\left(x^{0}\right)$ and $u(e) \geq H-O_{i}+\varphi$ in $B_{\varrho}\left(x^{0}\right)$. Hence for any $\vartheta \in C_{0}^{\infty}\left(B_{\varrho / 2}\left(x^{0}\right)\right)$ we may find $\varepsilon>0$ such that $u(e)+\varepsilon \vartheta \geq H-O_{i}+\varphi / 2$ in $B_{\varrho / 2}\left(x^{0}\right)$. As a consequence $v \equiv u(e)+\varepsilon \vartheta \in \mathcal{K}(H)$. Substituting this $v$ in (1.7), we find $v-u(e)=\varepsilon \vartheta$ and

$$
\int_{\Omega} H \operatorname{grad} u(e) \cdot \operatorname{grad} \vartheta d x \geq \int_{\Omega}(p-2 \omega H) \vartheta d x .
$$

Since the opposite inequality follows for $v \equiv u(e)-\varepsilon \vartheta$, we obtain

$$
\int_{\Omega}(H \operatorname{grad} u(e) \cdot \operatorname{grad} \vartheta-(p-2 \omega H) \vartheta) d x=0
$$

for all $\vartheta \in C_{0}^{\infty}\left(B_{\varrho / 2}\left(x^{0}\right)\right)$. Integrating by parts, we obtain (1.12) in $\Omega^{*} \backslash$ $Q(u(e))$.

Next, consider a point $x^{0} \in \Omega \backslash \overline{\Omega^{*}}$. We may find $B_{\varrho}\left(x^{0}\right) \subset \Omega \backslash \overline{\Omega^{*}}$ and for any $\vartheta \in C_{0}^{\infty}\left(B_{\underline{\varrho}}\left(x^{0}\right)\right)$ we substitute $v=u(e) \pm \vartheta$ in (1.7) to find that (1.12) holds in $\Omega \backslash \overline{\Omega^{*}}$.

Finally, integrating by parts and using (1.12), we may write

$$
\mathcal{L}_{\mathrm{TR}}(e, u(e))=\int_{\Omega} N_{z}(e, u(e)) \vartheta d x=\int_{Q(u(e))} N_{z}(e, u(e)) d x
$$

and the assertion of the lemma follows, since $\mathcal{Z}^{0}=0$ on $Q(u(e))$.

REMARK 1.1. Some results on the regularity of solutions to obstacle problems (cf. [7]) can justify the conjecture that $u(e) \in H^{2}(\Omega)$ provided $\Omega$ is convex and $H \in H^{2}\left(\Omega_{i}^{*}\right)$ for all $i=1, \ldots, N$. These assumptions seem to be sufficient for the justification of the functional $\mathcal{L}_{\mathrm{TR}}$.

Remark 1.2. Let $\theta \in X(\Omega)$. Then

$$
\mathcal{L}_{\mathrm{TR}}(e, u(e))=\int_{\Omega} \theta d \mu(e, u(e))=\int_{Q(u(e))} d \mu(e, u(e)) \geq 0,
$$

where $\mu(e, u(e))$ is a non-negative Radon measure with $\operatorname{supp} \mu(e, u(e)) \subset$ $Q(u(e))$.

Define

$$
\mathcal{R}(e) v=-\operatorname{div}(H \operatorname{grad} v)+\mathcal{Z}^{0}[v]^{-}
$$

for any $v \in H^{1}(\Omega)$. We can rewrite the inequality (1.7) for $v:=u(e)+\varphi$, where $\varphi \in X(\Omega), \varphi \geq 0$, as follows:

$$
\langle\mathcal{R}(e) u(e), \varphi\rangle \geq\langle p-2 \omega H, \varphi\rangle_{0} .
$$

As a consequence, $\mathcal{R}(e) u(e)-p+2 \omega H$ is a non-negative distribution on the domain $\Omega$, i.e., a non-negative Radon measure in $\Omega$ (cf. the Riesz-Schwartz Theorem in [8]). By virtue of (1.12), its support is contained in $Q(u(e))$ 
and (1.13) holds. This measure represents the interaction forces between the pseudoplate and the obstacles.

We define the following optimal design problems:

$$
\begin{gathered}
e_{\mathrm{DD}}^{*}=\underset{e \in U_{\mathrm{ad}}}{\arg \min } \mathcal{L}_{\mathrm{DD}}(u(e)), \\
e_{\mathrm{ISS}}^{*}=\underset{e \in U_{\mathrm{ad}}}{\arg \min } \mathcal{L}_{\mathrm{ISS}}(u(e)), \\
e_{\mathrm{COM}}^{*}=\underset{e \in U_{\mathrm{ad}}}{\arg \min } \mathcal{L}_{\mathrm{COM}}(e, u(e)) \\
e_{\mathrm{TR}}^{*}=\underset{e \in U_{\mathrm{ad}}}{\arg \min } \mathcal{L}_{\mathrm{TR}}(e, u(e)),
\end{gathered}
$$

where $u(e)$ denotes the solution of the state problem (1.7).

Moreover, we introduce the functionals

$$
F_{j}(v)=\left(\operatorname{meas} G_{j}\right)^{-1}\|\operatorname{grad} v\|_{0, G_{j}}^{2}-\tau^{2}, \quad j=1, \ldots, M,
$$

where $G_{j}$ are given subdomains of $\Omega$ and $\tau$ is a given constant. We define a set of admissible design variables

$$
\mathcal{S}_{\mathrm{ad}}=\left\{e \in U_{\mathrm{ad}}: \sum_{j=1}^{M}\left[F_{j}(u(e))\right]^{+}=0\right\}
$$

and the following weight minimization problem:

$$
e_{w}^{*}=\underset{e \in \mathcal{S}_{\mathrm{ad}}}{\arg \min } \int_{\Omega} \omega H d x .
$$

2. Existence of a solution to the optimal design problems. In this section we shall consider a class of abstract optimal control problems and prove their solvability. Then we shall apply the general result to our optimal design problems.

Let $U$ be a Banach space of controls, $U_{\text {ad }}$ a subset of admissible controls. We assume that $U_{\text {ad }}$ is compact in $U$. Let $V$ be a reflexive Banach space endowed with a norm $\|\cdot\|$ and let $V^{*}$ be its dual with a norm $\|\cdot\|_{*}$, the duality pairing between $V$ and $V^{*}$ being denoted by $\langle\cdot, \cdot\rangle$.

Definition 2.1. We say that a sequence $\left\{K_{n}\right\}, n=1,2, \ldots$, of convex subsets of $V$ converges to a set $K$, written

$$
K=\operatorname{Lim}_{n \rightarrow \infty} K_{n}
$$

if the following two conditions are satisfied:

(i) for any $v \in K$ there exists a sequence $\left\{v_{n}\right\}$ such that $v_{n} \in K_{n}$ and

$$
\lim _{n \rightarrow \infty} v_{n}=v \quad \text { in } V
$$

(ii) if $v_{n} \in K_{n}$ and $v_{n} \rightarrow v$ (weakly) in $V$, then $v \in K$. 
Definition 2.2 (cf. [6]). Let $\mathcal{W}_{n}: V \rightarrow[0,+\infty], n=1,2, \ldots$, be a sequence of functionals. We say that

$$
\mathcal{W}=\lim _{n \rightarrow \infty} \mathcal{W}_{n}
$$

if the following conditions hold:

(i) for each $v \in V$ there exists a sequence $\left\{v_{n}\right\}$ such that $v_{n} \in V$, $\lim _{n \rightarrow \infty} v_{n}=v$ in $V$ and

$$
\limsup _{n \rightarrow \infty} \mathcal{W}_{n}\left(v_{n}\right) \leq \mathcal{W}(v)
$$

(ii) for each subsequence $\left\{\mathcal{W}_{n_{k}}\right\}, k=1,2, \ldots$, and each sequence $\left\{v_{k}\right\}$, $v_{k} \in V$, weakly convergent to $v \in V$, we have

$$
\mathcal{W}(v) \leq \liminf _{k \rightarrow \infty} \mathcal{W}_{n_{k}}\left(v_{k}\right) .
$$

Remark 2.1. By Definition 2.2, $\mathcal{W}=\lim _{n \rightarrow \infty} \mathcal{W}_{n}$ implies that for each $v \in V$ there exists a sequence $\left\{v_{n}\right\}$ such that $v_{n} \in V, v_{n} \rightarrow v$ in $V$ and $\lim _{n \rightarrow \infty} \mathcal{W}_{n}\left(v_{n}\right)=\mathcal{W}(v)$.

Consider a system $\{\mathcal{K}(e)\}, e \in U_{\text {ad }}$, of closed convex subsets $\mathcal{K}(e) \subset V$ and a family $\{A(e)\}, e \in U_{\mathrm{ad}}$, of operators $A(e): V \rightarrow V^{*}$ satisfying the following assumptions:

(i) $\bigcap_{e \in U_{\text {ad }}} \mathcal{K}(e) \neq \emptyset$.

(ii) $e_{n} \rightarrow e$ in $U, e_{n} \in U_{\text {ad }} \Rightarrow \mathcal{K}(e)=\operatorname{Lim}_{n \rightarrow \infty} \mathcal{K}\left(e_{n}\right)$.

(iii) There exist constants $0<\alpha_{A}<M_{A}$ independent of $e \in U_{\text {ad }}$ and such that

$$
\begin{gathered}
\alpha_{A}\|v-w\|^{2} \leq\langle A(e) v-A(e) w, v-w\rangle, \\
\|A(e) v-A(e) w\|_{*} \leq M_{A}\|v-w\|,
\end{gathered}
$$

for all $v, w \in V$ and all $e \in U_{\text {ad }}$.

(iv) $e_{n} \rightarrow e$ in $U, e_{n} \in U_{\text {ad }} \Rightarrow A\left(e_{n}\right) v \rightarrow A(e) v$ in $V^{*}$ for all $v \in V$.

Moreover, we consider a system $\{\Phi(e)\}, e \in U_{\mathrm{ad}}$, of functionals $\Phi(e)$ : $V \rightarrow[0,+\infty]$, lower semicontinuous and convex on $V$ and such that

$$
\begin{gathered}
e_{n} \in U_{\mathrm{ad}}, e_{n} \rightarrow e \text { in } U \Rightarrow \Phi(e)=\lim \Phi\left(e_{n}\right), \\
\operatorname{dom} \Phi(e) \equiv\{v \in V: \Phi(e) v<+\infty\}=\mathcal{K}(e)
\end{gathered}
$$

for all $e \in U_{\text {ad }}$.

Furthermore, we assume that for each sequence $\left\{e_{n}\right\}, n=1,2, \ldots$, such that $e_{n} \in U_{\text {ad }}, e_{n} \rightarrow e$ in $U$, there is a bounded sequence $\left\{a_{n}\right\}$ with $a_{n} \in$ $\mathcal{K}\left(e_{n}\right)$, and

$$
\limsup _{n \rightarrow \infty} \Phi\left(e_{n}\right) a_{n}<+\infty .
$$

Finally, let a functional $f \in V^{*}$ and a continuous mapping $B: U \rightarrow V^{*}$ be given. 
For any $e \in U_{\text {ad }}$ consider the following variational inequality: find $u(e) \in$ $\mathcal{K}(e)$ such that

$$
\langle A(e) u(e), v-u(e)\rangle+\Phi(e) v-\Phi(e) u(e) \geq\langle f+B(e), v-u(e)\rangle
$$

for all $v \in \mathcal{K}(e)$.

There exists a unique solution $u(e)$ for any $e \in U_{\text {ad }}$. In fact, we can employ the general theory of variational inequalities (cf. e.g. [5, Thm. 8.5] for a proof of existence; the uniqueness follows from the strong monotonicity).

Next, let $\mathcal{L}: U \times V \rightarrow \mathbb{R}$ be a functional such that

$$
\begin{aligned}
e_{n} \in U_{\mathrm{ad}}, e_{n} \rightarrow e \text { in } U \text { and } v_{n} \rightarrow & v \text { (weakly) in } V \\
& \Rightarrow \liminf _{n \rightarrow \infty} \mathcal{L}\left(e_{n}, v_{n}\right) \geq \mathcal{L}(e, v) .
\end{aligned}
$$

Let us introduce a functional $J: U_{\text {ad }} \rightarrow \mathbb{R}$ by the formula

$$
J(e)=\mathcal{L}(e, u(e)),
$$

where $u(e)$ is the solution of the problem (2.4). We shall solve the optimization problem

$$
e^{*}=\underset{e \in U_{\text {ad }}}{\arg \min } J(e) .
$$

THeOREm 2.1. Let the data of the state problem (2.4) satisfy the assumptions (2.1)-(2.3). Let $e_{n} \in U_{\mathrm{ad}}, e_{n} \rightarrow e^{*}$ in $U$. Then $u\left(e_{n}\right) \rightarrow u\left(e^{*}\right)$ in $V$.

Proof. For brevity, write $u_{n} \equiv u\left(e_{n}\right), A_{n}=A\left(e_{n}\right), \Phi_{n}=\Phi\left(e_{n}\right), \mathcal{K}_{n}=$ $\mathcal{K}\left(e_{n}\right), B_{n}=B\left(e_{n}\right)$. Consider the inequality (2.4) for any $e_{n}, n=1,2, \ldots$ Inserting $v=a_{n}$ (see (2.3)), and adding the term $\left\langle A_{n} a_{n}, u_{n}-a_{n}\right\rangle$ to both sides, we derive the inequality

$$
\begin{aligned}
& \left\langle A_{n} u_{n}-A_{n} a_{n}, u_{n}-a_{n}\right\rangle+\Phi_{n} u_{n} \\
& \leq\left\langle f+B_{n}, u_{n}-a_{n}\right\rangle+\left\langle A_{n} a_{n}, a_{n}-u_{n}\right\rangle+\Phi_{n} a_{n} .
\end{aligned}
$$

Using (2.1)(iii)\&(iv), (2.3) and the continuity of $B$, we deduce that $\left\|u_{n}\right\| \leq C$ for all $n$. Hence there exists a subsequence $\left\{u_{k}\right\} \subset\left\{u_{n}\right\}$ and $u^{*} \in V$ such that $u_{k} \rightarrow u^{*}$ (weakly) in $V$. The assumption (2.1)(ii) implies that $u^{*} \in$ $\mathcal{K}\left(e^{*}\right)$, so that

$$
\Phi\left(e^{*}\right) u^{*}<+\infty
$$

by (2.2). Using Remark 2.1, we can find a sequence $\left\{\theta_{k}\right\}$ such that $\theta_{k} \in \mathcal{K}\left(e_{k}\right)$ and

$$
\theta_{k} \rightarrow u^{*} \text { in } V, \quad \lim \Phi_{k} \theta_{k}=\Phi\left(e^{*}\right) u^{*} .
$$

Note that $\theta_{k} \in \mathcal{K}\left(e_{k}\right)$ follows from (2.2) and (2.8), (2.9). 
Next, consider again the inequality (2.4) for $e_{k}$, insert $v:=\theta_{k}$, and add the term $\left\langle A_{k} \theta_{k}, u_{k}-\theta_{k}\right\rangle$ to both sides to obtain

$$
\begin{gathered}
\limsup \left\langle A_{k} u_{k}-A_{k} \theta_{k}, u_{k}-\theta_{k}\right\rangle \\
\leq \limsup \left\langle A_{k} \theta_{k}, \theta_{k}-u_{k}\right\rangle+\lim \sup \left\langle f+B_{k}, u_{k}-\theta_{k}\right\rangle \\
\quad+\limsup \Phi_{k} \theta_{k}-\liminf \Phi_{k} u_{k} \leq 0 .
\end{gathered}
$$

The last inequality follows from the weak convergence of $u_{k},(2.9)$, the continuity of $B$, the assumption (2.2) and the following assertion:

$$
\begin{aligned}
& e_{k} \rightarrow e, e_{k} \in U_{\text {ad }} \text { and } v_{k} \rightarrow v \text { in } V \\
& \quad \Rightarrow\left\|A_{k} v_{k}-A(e) v\right\|_{*} \leq M_{A}\left\|v_{k}-v\right\|+\left\|A_{k} v-A(e) v\right\|_{*} \rightarrow 0,
\end{aligned}
$$

which is a consequence of (2.1)(iii)\&(iv).

Using (2.1)(iii) and (2.10), we obtain $\lim \left\|u_{k}-\theta_{k}\right\|=0$. Combining this with $(2.9)$, we arrive at

$$
u_{k} \rightarrow u^{*} \text { in } V \text {. }
$$

Moreover, (2.11) and (2.12) imply

$$
A_{k} u_{k} \rightarrow A\left(e^{*}\right) u^{*} \text { in } V^{*} \text {. }
$$

Given any $v \in \mathcal{K}\left(e^{*}\right)$, by $(2.2)$ and Remark 2.1 there exists a sequence $\left\{\varphi_{k}\right\}$ such that $\varphi_{k} \in \mathcal{K}\left(e_{k}\right), \varphi_{k} \rightarrow v$ in $V$ and

$$
\Phi_{k} \varphi_{k} \rightarrow \Phi\left(e^{*}\right) v
$$

Inserting $v:=\varphi_{k}$ in (2.4) for $e_{k}$, we obtain

$$
\left\langle A_{k} u_{k}, u_{k}-\varphi_{k}\right\rangle-\left\langle f+B_{k}, u_{k}-\varphi_{k}\right\rangle \leq \Phi_{k} \varphi_{k}-\Phi_{k} u_{k} .
$$

Passing to the limsup on both sides and using (2.12)-(2.14) and the assumption $(2.2)$, we arrive at

$$
\left\langle A\left(e^{*}\right), u^{*}-v\right\rangle-\left\langle f+B\left(e^{*}\right), u^{*}-v\right\rangle \leq \Phi\left(e^{*}\right) v-\Phi\left(e^{*}\right) u^{*} .
$$

From the uniqueness of $u\left(e^{*}\right)$, we deduce $u^{*}=u\left(e^{*}\right)$. Moreover, the whole sequence $\left\{u\left(e_{n}\right)\right\}$ converges to $u\left(e^{*}\right)$ in $V$.

THEOREM 2.2. Let the data of the state problem (2.4) satisfy the assumptions (2.1)-(2.3). Let the functional $\mathcal{L}$ satisfy the condition (2.5). Then there exists at least one solution of the optimal control problem (2.6).

Proof. Since the set $U_{\mathrm{ad}}$ is compact in $U$, there exists a sequence $\left\{e_{n}\right\}$ such that $e_{n} \in U_{\text {ad }}$ and

$$
e_{n} \rightarrow e^{*} \quad \text { in } U, \quad e^{*} \in U_{\text {ad }}, \quad J\left(e_{n}\right) \rightarrow \inf _{e \in U_{\text {ad }}} J(e) .
$$

Then (2.5) and Theorem 2.1 imply that

$$
\mathcal{L}\left(e^{*}, u\left(e^{*}\right)\right) \leq \liminf _{n \rightarrow \infty} \mathcal{L}\left(e_{n}, u\left(e_{n}\right)\right)=\inf _{e \in U_{\text {ad }}} \mathcal{L}(e, u(e)) .
$$

As a consequence, $e^{*}$ is a solution to the problem (2.6). 
Let us apply Theorem 2.2 to the proof of existence of solutions to the optimal design problems (1.14)-(1.17). We have to verify the assumptions of Theorem 2.1.

We have $U=C(\bar{\Omega}) \times C\left(\bar{\Omega} \backslash \Omega^{*}\right) \times C\left(\bar{\partial}_{C}\right)$ and

$$
U_{\mathrm{ad}}=U_{\mathrm{ad}}^{H} \times U_{\mathrm{ad}}^{\mathcal{Z}} \times U_{\mathrm{ad}}^{\mathcal{F}} .
$$

Lemma 2.2. For any $H \in U_{\text {ad }}^{H}$ the set $\mathcal{K}(H)$, defined in (1.3), is a closed and convex subset of $V$ and

$$
H_{n} \in U_{\text {ad }}^{H}, H_{n} \rightarrow H \text { in } C(\bar{\Omega}) \Rightarrow \mathcal{K}(H)=\operatorname{Lim}_{n \rightarrow \infty} \mathcal{K}\left(H_{n}\right) .
$$

Moreover, $v \equiv 0 \in \bigcap \mathcal{K}(H)$ for all $H \in U_{\text {ad }}^{H}$.

Proof. The closedness follows from the Lebesgue Theorem. The convexity is immediate. Let $H_{n} \in U_{\text {ad }}^{H}, H_{n} \rightarrow H$ in $C(\bar{\Omega})$. There exists a function $\vartheta \in C_{0}(\Omega)$ such that $0 \leq \vartheta \leq 1$ in $\Omega$ and $\vartheta=1$ for $x \in \Omega^{*}$. For any $v \in \mathcal{K}(H)$ we construct a sequence

$$
v_{n}=v+\vartheta\left\|H_{n}-H\right\|_{\infty}
$$

Then

$$
v_{n} \geq H-O_{i}+\left(H_{n}-H\right)=H_{n}-O_{i}
$$

for a.a. $x \in \Omega_{i}^{*}$, so that $v_{n} \in \mathcal{K}\left(H_{n}\right)$. Moreover,

$$
\left\|v_{n}-v\right\|_{1}=\left\|H_{n}-H\right\|_{\infty}\|\vartheta\|_{1} \rightarrow 0 \quad \text { as } n \rightarrow \infty .
$$

Next, let $v_{n} \in \mathcal{K}\left(H_{n}\right), v_{n} \rightarrow v$ in $V$. By the Rellich Theorem, we have $v_{n} \rightarrow v$ in $L_{2}(\Omega)$ and $v_{n} \geq H_{n}-O_{i}$ for a.a. $x \in \Omega_{i}^{*}$. The Lebesgue Theorem yields $v \geq H-O_{i}$, so that $v \in \mathcal{K}(H)$.

Since $O_{i} \geq H_{\max }$ for all $i=1, \ldots, N$ by assumption (1.1), the zero function belongs to $\mathcal{K}(H)$ for any $H \in U_{\text {ad }}$.

As a consequence, (2.1)(i)\&(ii) are satisfied. For any $e \in U_{\text {ad }}$ we define the mapping $A(e): V \rightarrow V^{*}$ by the formula

$$
\langle A(e) v, w\rangle=a(H ; v, w)+b(\mathcal{Z} ; v, w) \quad \forall v, w \in V
$$

Lemma 2.3. The family of operators $\{A(e)\}, e \in U_{\text {ad }}$, satisfies the assumptions (2.1)(iii)\&(iv).

Proof. It is readily seen that

$$
a(H ; v, v) \geq H_{\min } \int_{\Omega}|\operatorname{grad} v|^{2} d x \geq C_{F} H_{\min }\|v\|_{1}^{2}
$$

for all $v \in V$, since we can employ the Friedrichs inequality. We also have

$$
b(\mathcal{Z} ; v, v-z)-b(\mathcal{Z} ; z, v-z)=\int_{\Omega \backslash \Omega^{*}} \mathcal{Z}\left([v]^{-}-[z]^{-}\right)(v-z) d x \geq 0,
$$


since

$$
\left([v]^{-}-[z]^{-}\right)(v-z) \geq\left([v]^{-}-[z]^{-}\right)^{2} .
$$

Combining (2.16), (2.17) and (2.15), we may write

$$
\begin{aligned}
\langle A(e) v-A(e) z, v-z\rangle & =a(H ; v-z, v-z)+b(\mathcal{Z} ; v, v-z)-b(\mathcal{Z} ; z, v-z) \\
& \geq C_{F} H_{\min }\|v-z\|_{1}^{2}
\end{aligned}
$$

for all $H \in U_{\text {ad }}^{H}, \mathcal{Z} \in U_{\text {ad }}^{\mathcal{Z}}, v \in V$ and $z \in V$.

Next, we have

$$
\begin{aligned}
|a(H ; v, w)-a(H ; z, w)| & =\left|\int_{\Omega} H \operatorname{grad}(v-z) \operatorname{grad} w d x\right| \\
& \leq H_{\max }\|v-z\|_{1}\|w\|_{1}
\end{aligned}
$$

and

$$
\begin{aligned}
|b(\mathcal{Z} ; v, w)-b(\mathcal{Z} ; z, w)| & =\left|\int_{\Omega \backslash \Omega^{*}} \mathcal{Z}\left([v]^{-}-[z]^{-}\right) w d x\right| \\
& \leq \mathcal{Z}_{\max }\|v-z\|_{1}\|w\|_{1}
\end{aligned}
$$

where the relation $\left|[v]^{-}-[z]^{-}\right| \leq|v-z|$ has been used. Combining (2.15), (2.18) and (2.19), we obtain

$$
|\langle A(e) v-A(e) z, w\rangle| \leq\left(H_{\max }+\mathcal{Z}_{\max }\right)\|v-z\|_{1}\|w\|_{1} .
$$

As a consequence, the assumption (2.1)(iii) is satisfied. To verify (2.1)(iv), we write

$$
\begin{aligned}
\left|\left\langle A\left(e_{n}\right) v-A(e) v, w\right\rangle\right| \leq & \left|\int_{\Omega}\left(H_{n}-H\right) \operatorname{grad} v \cdot \operatorname{grad} w d x\right| \\
& +\left|\int_{\Omega \backslash \Omega^{*}}\left(\mathcal{Z}_{n}-\mathcal{Z}\right)[v]^{-} w d x\right| \\
\leq & \left(\left\|H_{n}-H\right\|_{\infty}+\left\|\mathcal{Z}_{n}-\mathcal{Z}\right\|_{\infty}\right)\left\|v_{1}\right\| \cdot\|w\|_{1},
\end{aligned}
$$

using the relation $\left|[v]^{-}\right| \leq|v|$. Then

$$
\left\|A\left(e_{n}\right) v-A(e) v\right\|_{*} \leq\left(\left\|H_{n}-H\right\|_{\infty}+\left\|\mathcal{Z}_{n}-\mathcal{Z}\right\|_{\infty}\right)\|v\|_{1} \rightarrow 0
$$

if $e_{n} \rightarrow e$ in $U$.

LEMma 2.4. The system $\{\Phi(e)\}, e \in U_{\mathrm{ad}}$, of functionals defined by (1.6) satisfies the assumptions (2.2) and (2.3).

Proof. Since the integral is continuous on $V$ and the indicatrix is lower semicontinuous, their sum is lower semicontinuous on $V$ for any $H \in U_{\text {ad }}^{H}$ and $\mathcal{F} \in U_{\text {ad }}^{\mathcal{F}}$. The convexity is immediate. For any $e \in U_{\text {ad }}$ and $v \in \mathcal{K}(H)$, the integral is finite and the indicatrix vanishes. 
Let us verify the assumption (2.2). Let us write

$$
\begin{gathered}
\Phi(e) v=\Phi_{1}(e) v+\Phi_{2}(e) v, \\
\Phi_{1}(e) v=\int_{\partial \Omega_{C}} \mathcal{F}|v| d s, \quad \Phi_{2}(e) v=I_{\mathcal{K}(H)}(v),
\end{gathered}
$$

$\left|\Phi\left(e_{n}\right) v_{n}-\Phi(e) v\right| \leq\left|\Phi_{1}\left(e_{n}\right) v_{n}-\Phi_{1}(e) v\right|+\left|\Phi_{2}\left(e_{n}\right) v_{n}-\Phi_{2}(e) v\right|=\left|\Lambda_{1}\right|+\left|\Lambda_{2}\right|$.

We verify the conditions (i) and (ii) of Definition 2.2. First, let $v \in \mathcal{K}(H)$. By Lemma 2.2 there exists a sequence $\left\{v_{n}\right\}$ such that $v_{n} \in \mathcal{K}\left(H_{n}\right)$ and $v_{n} \rightarrow v$ in $V$. Then we have

$$
\begin{aligned}
\left|\Lambda_{1}\right| & \leq\left|\Phi_{1}\left(e_{n}\right) v_{n}-\Phi_{1}(e) v_{n}\right|+\left|\Phi_{1}(e) v_{n}-\Phi_{1}(e) v\right| \\
& \leq \int_{\partial \Omega_{C}}\left(\left|\mathcal{F}_{n}-\mathcal{F}\right| \cdot\left|v_{n}\right|+\mathcal{F}\left|v_{n}-v\right|\right) d s \\
& \leq C\left(\left\|\mathcal{F}_{n}-\mathcal{F}\right\|_{\infty}\left\|v_{n}\right\|_{1}+\mathcal{F}_{\max }\left\|v_{n}-v\right\|_{1}\right) \rightarrow 0, \\
\Lambda_{2} & =I_{\mathcal{K}\left(H_{n}\right)}\left(v_{n}\right)-I_{\mathcal{K}(H)}(v)=0 .
\end{aligned}
$$

Altogether, we obtain

$$
\lim \Phi\left(e_{n}\right) v_{n}=\Phi(e) v .
$$

Second, let $v \notin \mathcal{K}(H)$. Setting $v_{n}=v$ for all $n=1,2, \ldots$, we have

$$
\begin{aligned}
\limsup \Phi\left(e_{n}\right) v_{n} & \leq \limsup \int_{\partial \Omega_{C}} \mathcal{F}_{n}|v| d s+\limsup I_{\mathcal{K}\left(H_{n}\right)}(v) \\
& \leq \int_{\partial \Omega_{C}} \mathcal{F}|v| d s+\infty=\Phi_{1}(e) v+\Phi_{2}(e)(v) \\
& =\Phi(e) v
\end{aligned}
$$

since $I_{\mathcal{K}(H)}(v)=+\infty$.

Combining (2.20) and (2.21), we obtain $\lim \sup \Phi\left(e_{n}\right) v_{n} \leq \Phi(e) v$ for any $v \in V$. As a consequence, condition (i) is satisfied.

Next, let $v_{n} \rightarrow v$ in $V$. We have

$$
\liminf \Phi\left(e_{n}\right) v_{n} \geq \liminf \Phi_{1}\left(e_{n}\right) v_{n}+\liminf \Phi_{2}\left(e_{n}\right) v_{n}
$$

Using the compactness of the trace mapping $H^{1}(\Omega) \rightarrow L_{2}(\partial \Omega)$, we may write

$$
\left|\Phi_{1}\left(e_{n}\right) v_{n}-\Phi_{1}(e) v\right| \leq \int_{\partial \Omega_{C}}\left(\left|\mathcal{F}_{n}-\mathcal{F}\right| \cdot\left|v_{n}\right|+\mathcal{F}\left|v_{n}-v\right|\right) d s \rightarrow 0,
$$

so that

$$
\lim \Phi_{1}\left(e_{n}\right) v_{n}=\Phi_{1}(e) v
$$

We have

$$
\liminf \Phi_{2}\left(e_{n}\right) v_{n}=\liminf I_{\mathcal{K}\left(H_{n}\right)}\left(v_{n}\right)=a
$$


where $a$ is either $+\infty$ or zero. If $a=+\infty$, then obviously

$$
a \geq I_{K(H)}(v) .
$$

If $a=0$, there exists a subsequence $\left\{v_{k}\right\} \subset\left\{v_{n}\right\}$ such that $v_{k} \in \mathcal{K}\left(H_{k}\right)$ for all $k \rightarrow \infty$. By Lemma 2.2 the weak limit $v$ belongs to $\mathcal{K}(H)$, so that $I_{\mathcal{K}(H)}(v)=0$ and $(2.22)$ holds again. As a consequence, condition (ii) is fulfilled, as well. Thus $\Phi(e)=\lim \Phi\left(e_{n}\right)$ whenever $e_{n} \rightarrow e$ in $U$.

To verify condition (2.3), we can set $a_{n}=0, n=1,2, \ldots$, since $0 \in \mathcal{K}(H)$ for all $H \in U_{\text {ad }}^{H}$, by (1.1). Then $\Phi\left(e_{n}\right) a_{n}=0$ for all $n$.

Lemma 2.5. Any of the cost functionals (1.8)-(1.11) satisfies the assumption (2.5).

Proof. Let $e_{n} \in U_{\mathrm{ad}}, e_{n} \rightarrow e$ in $U$ and $v_{n} \rightarrow v$ in $V$. By the Rellich Theorem, $v_{n} \rightarrow v$ in $L_{2}(\Omega)$, so that

$$
\left|\mathcal{L}_{\mathrm{DD}}\left(v_{n}\right)-\mathcal{L}_{\mathrm{DD}}(v)\right| \leq \int_{\Omega}\left|\left(v_{n}-z\right)^{2}-(v-z)^{2}\right| d x \leq\left\|v_{n}+v-2 z\right\|_{0}\left\|v_{n}-v\right\|_{0} \rightarrow 0
$$

and so

$$
\lim _{n \rightarrow \infty} \mathcal{L}_{\mathrm{DD}}\left(v_{n}\right)=\mathcal{L}_{\mathrm{DD}}(v) .
$$

The functional $\mathcal{L}_{\text {ISS }}$ is convex and differentiable in $V$. Therefore it is weakly lower semicontinuous and (2.5) follows.

Next, we may write

$$
\mathcal{L}_{\mathrm{COM}}\left(e_{n}, v_{n}\right)=\left\langle p-2 \omega H_{n}, v_{n}\right\rangle_{0}=\left\langle p-2 \omega H, v_{n}\right\rangle_{0}+\psi_{n},
$$

where

$$
\left|\psi_{n}\right|=2 \omega\left|\left\langle H-H_{n}, v_{n}\right\rangle_{0}\right| \leq 2 \omega\left\|H-H_{n}\right\|_{\infty} C\left\|v_{n}\right\|_{1} \rightarrow 0 .
$$

Then

$$
\lim \mathcal{L}_{\mathrm{COM}}\left(e_{n}, v_{n}\right)=\langle p-2 \omega H, v\rangle_{0}=\mathcal{L}_{\mathrm{COM}}(e, v) .
$$

Consider a fixed $\theta \in X(\Omega)$ in (1.11) and write

$$
\begin{aligned}
\mathcal{L}_{\mathrm{TR}}\left(e_{n}, v_{n}\right)=\mathcal{L}_{\mathrm{TR}}\left(e, v_{n}\right)+M_{n}, \\
\left|M_{n}\right|=\mid \int_{\Omega}\left[\left(H_{n}-H\right) \operatorname{grad} v_{n} \cdot \operatorname{grad} \theta\right. \\
\left.+2 \omega\left(H_{n}-H\right) \theta+\left(\mathcal{Z}_{n}^{0}-\mathcal{Z}^{0}\right)\left[v_{n}\right]^{-} \theta\right] d x \mid \\
\leq C\left[\left\|H_{n}-H\right\|_{\infty}\left(\left\|v_{n}\right\|_{1}+1\right)+\left\|\mathcal{Z}_{n}^{0}-\mathcal{Z}^{0}\right\|_{\infty}\left\|v_{n}\right\|_{1}\right] \rightarrow 0 .
\end{aligned}
$$

By the Rellich Theorem,

$$
\left|\int_{\Omega} \mathcal{Z}^{0}\left(\left[v_{n}\right]^{-}-[v]^{-}\right) \theta d x\right| \leq \mathcal{Z}_{\max } \int_{\Omega}\left|v_{n}-v\right| \theta d x \leq C\left\|v_{n}-v\right\|_{0} \rightarrow 0 .
$$


As a consequence, we obtain

$$
\lim \mathcal{L}_{\mathrm{TR}}\left(e, v_{n}\right)=\mathcal{L}_{\mathrm{TR}}(e, v) .
$$

Combining (2.23)-(2.25), we arrive at $\lim \mathcal{L}_{\mathrm{TR}}\left(e_{n}, v_{n}\right)=\mathcal{L}_{\mathrm{TR}}(e, v)$.

The Arzelà-Ascoli Theorem yields the compactness of the sets $U_{\text {ad }}^{H}, U_{\text {ad }}^{\mathcal{Z}}$ and $U_{\text {ad }}^{\mathcal{F}}$ in the spaces $C(\bar{\Omega}), C\left(\bar{\Omega} \backslash \Omega^{*}\right)$ and $C\left(\bar{\partial}_{C}\right)$, respectively. Thus $U_{\text {ad }}$ is compact in $U$. Finally, setting

$$
\langle f, v\rangle=\langle p, v\rangle, \quad\langle B e, v\rangle=-2 \omega\langle H, v\rangle_{0},
$$

it is readily seen that $f \in V^{*}$ and $B: U \rightarrow V^{*}$ is a continuous mapping.

Altogether, all the assumptions of Theorem 2.2 are fulfilled, by Lemmas $2.2-2.5$. As a consequence, each of the optimal design problems (1.14)-(1.17) has at least one solution.

3. Existence of a solution to the weight minimization problem. The existence of a solution to the problem (1.18) will be proved by means of a penalization method. Consider a penalized cost functional

$$
\mathcal{L}_{w}(\varepsilon, e, u)=\langle\omega, H\rangle_{0}+\varepsilon^{-1} \sum_{j=1}^{M}\left[F_{j}(u)\right]^{+},
$$

where $\varepsilon$ is an arbitrary positive constant parameter. We define the following penalized optimization problem: find

$$
e_{\varepsilon}=\underset{e \in U_{\mathrm{ad}}}{\arg \min } \mathcal{L}_{w}(\varepsilon, e, u(e)),
$$

where $u(e)$ is the solution of the state problem (1.7).

Lemma 3.1. Let $e_{n} \rightarrow e$ in $U, e_{n} \in U_{\mathrm{ad}}$, as $n \rightarrow \infty$. Then

$$
\lim _{n \rightarrow \infty}\left[F_{j}\left(u\left(e_{n}\right)\right)\right]^{+}=\left[F_{j}(u(e))\right]^{+}, \quad j=1, \ldots, M .
$$

Proof. We may write

$$
\begin{aligned}
\mid\left[F_{j}\left(u\left(e_{n}\right)\right)\right]^{+} & -\left[F_{j}(u(e))\right]^{+} \mid \\
& \leq\left|F_{j}\left(u\left(e_{n}\right)\right)-F_{j}(u(e))\right| \\
& \leq\left.\left(\operatorname{meas} G_{j}\right)^{-1} \int_{G_{j}}|| \operatorname{grad} u\left(e_{n}\right)\right|^{2}-|\operatorname{grad} u(e)|^{2} \mid d x \\
& \leq C \int_{G_{j}}\left|\operatorname{grad}\left(u\left(e_{n}\right)-u(e)\right)\right|\left(\left|\operatorname{grad} u\left(e_{n}\right)\right|+|\operatorname{grad} u(e)|\right) d x \\
& \leq C\left\|u\left(e_{n}\right)-u(e)\right\|_{1}\left(\left\|u\left(e_{n}\right)\right\|_{1}+\|u(e)\|_{1}\right) \rightarrow 0,
\end{aligned}
$$

where Theorem 2.1 has been used in the last step. 
LEMma 3.2. The penalized optimization problem (3.1) has a solution for any positive $\varepsilon$.

Proof. By Lemma 3.1, the functionals $e \mapsto\left[F_{j}(u(e))\right]^{+}$are continuous in $U_{\text {ad }}$ and so is the weight $\langle\omega, H\rangle_{0}$. Since the set $U_{\text {ad }}$ is compact in $U$, there exists a minimizer in $U_{\text {ad }}$.

TheOREM 3.1. Assume that $\mathcal{S}_{\mathrm{ad}} \neq \emptyset$ and the data satisfy all assumptions of Theorem 2.1. Let $\left\{e_{\varepsilon_{n}}\right\}, \varepsilon_{n} \rightarrow 0_{+}$, be a sequence of solutions of the penalized optimization problems (3.1). Let $\left\{u\left(e_{\varepsilon_{n}}\right)\right\}$ be the corresponding sequence of solutions to the state problem (1.7). Then there exists a subsequence $\left\{e_{\varepsilon_{k}}\right\} \subset\left\{e_{\varepsilon_{n}}\right\}$ and $e_{w}^{*} \in \mathcal{S}_{\text {ad }}$ such that

$$
\begin{array}{cl}
e_{\varepsilon_{k}} \rightarrow e_{w}^{*} & \text { in } U, \\
u\left(e_{\varepsilon_{k}}\right) \rightarrow u\left(e_{w}^{*}\right) & \text { in } V,
\end{array}
$$

where $e_{w}^{*}$ is a solution to the weight minimization problem (1.18).

Proof. Since $U_{\mathrm{ad}}$ is compact in $U$, there exists a subsequence such that (3.2) holds. Then (3.3) follows from Theorem 2.1. Let us show that $e_{w}^{*}$ is a solution of (1.18). By definition, setting

$$
J_{w}(e)=\langle\omega, H\rangle_{0},
$$

we have

$$
J_{w}\left(e_{\varepsilon_{k}}\right)+\varepsilon_{k}^{-1} \sum_{j=1}^{M}\left[F_{j}\left(u\left(e_{\varepsilon_{k}}\right)\right)\right]^{+} \leq J_{w}(e)
$$

for any $e \in \mathcal{S}_{\text {ad }}$. Then

$$
\sum_{j=1}^{M}\left[F_{j}\left(u\left(e_{k}\right)\right)\right]^{+} \leq \varepsilon_{k} J_{w}(e) .
$$

Passing to the limit as $\varepsilon_{k} \rightarrow 0_{+}$and using Lemma 3.1, we obtain

$$
\sum_{j=1}^{M}\left[F_{j}\left(u\left(e_{w}^{*}\right)\right)\right]^{+} \leq 0
$$

so that $e_{w}^{*} \in \mathcal{S}_{\text {ad }}$ follows.

Next, in view of (3.4) we have

$$
J_{w}\left(e_{\varepsilon_{k}}\right) \leq J_{w}(e)
$$

for all $e \in \mathcal{S}_{\text {ad }}$. Pass to the limit, use (3.2) and the continuity of $J_{w}$ in $U$ to obtain

$$
J_{w}\left(e_{w}^{*}\right) \leq J_{w}(e)
$$

for any $e \in \mathcal{S}_{\text {ad }}$. Hence $e_{w}^{*}$ is a solution of (1.18). 
COROLlary 3.1. Assume that $\mathcal{S}_{\mathrm{ad}} \neq \emptyset$ and the data satisfy all assumptions of Theorem 2.1. Then there exists at least one solution of the weight minimization problem (1.18).

Proof. This is a consequence of Lemma 3.2 and Theorem 3.1.

4. Reliable solutions of problems with uncertain data. In this section we will consider the state problem (1.7) with some uncertain input data. It may happen that the distributed load $p$, the coefficient $\mathcal{Z}$ of the elastic foundation and the slip limit $\mathcal{F}$ are uncertain, i.e., they are not given uniquely, but the only available information is that they belong to some given sets $U_{\mathrm{ad}}^{p}, U_{\mathrm{ad}}^{\mathcal{Z}}$ and $U_{\mathrm{ad}}^{\mathcal{F}}$, respectively. On the other hand, we assume that the half-thickness function $H$ is fixed, $H \in C^{(0), 1}(\bar{\Omega})$ and $H>0$.

Let $U_{\text {ad }}^{\mathcal{Z}}$ and $U_{\text {ad }}^{\mathcal{F}}$ be the sets defined in Section 1 . Let $\Omega$ be decomposed into $M$ disjoint subdomains, i.e.,

$$
\bar{\Omega}=\bigcup_{m=1}^{M} \bar{\Omega}_{m}, \quad \Omega_{m} \cap \Omega_{k}=\emptyset \text { if } m \neq k,
$$

and let

$$
\begin{aligned}
& U_{\mathrm{ad}}^{p}=\left\{p \in L^{\infty}(\Omega):\left.p\right|_{\bar{\Omega}_{m}} \in C^{(0), 1}\left(\bar{\Omega}_{m}\right), m=1, \ldots, M,\right. \\
&\left.\left\|p-p_{0}\right\|_{\infty} \leq C_{1},\left\|\frac{\partial p}{\partial x_{i}}\right\|_{\infty} \leq C_{2}, i=1,2\right\},
\end{aligned}
$$

where $p_{0}$ is a given function such that

$$
\left.p_{0}\right|_{\bar{\Omega}_{m}} \in C^{(0), 1}\left(\bar{\Omega}_{m}\right), \quad\left\|\frac{\partial p_{0}}{\partial x_{i}}\right\|_{\infty} \leq C_{2}, \quad i=1,2
$$

and $C_{1}, C_{2}$ are given constants.

Note that any $p \in U_{\mathrm{ad}}^{p}$ is a piecewise Lipschitz-continuous function which does not differ "too much" from a "central" piecewise Lipschitz-continuous function $p_{0}$.

We introduce the set

$$
U_{\mathrm{ad}}=U_{\mathrm{ad}}^{p} \times U_{\mathrm{ad}}^{\mathcal{Z}} \times U_{\mathrm{ad}}^{\mathcal{F}},
$$

the space

$$
U=\left(\prod_{m=1}^{M} C\left(\bar{\Omega}_{m}\right)\right) \times C\left(\bar{\Omega} \backslash \Omega^{*}\right) \times C\left(\partial \bar{\Omega}_{C}\right)
$$

and the triples of data

$$
e \equiv\{p, \mathcal{Z}, \mathcal{F}\} \in U_{\text {ad }} .
$$

We shall employ the method of reliable solutions alias worst scenario method (see [2], [3]), which consists of the following steps: 
(i) choose a functional-criterion $\{e, u\} \mapsto \Phi(e, u)$,

(ii) solve the following maximization problem:

$$
e^{*}=\underset{e \in U_{\mathrm{ad}}}{\arg \max } \Phi(e, u(e)),
$$

where $u(e)$ denotes the (unique) solution of the state problem (1.7) for the input data $e$.

The choice of the criterion $\Phi$ depends on the technical demands. For instance, it can represent maximal deflection of the pseudoplate, maximal intensity of shear stresses or the resultant transverse reactive force on a given inner obstacle. Thus we can define

$$
\begin{aligned}
& \Phi_{1}(u)=\max _{1 \leq j \leq J}\left(\operatorname{meas} G_{j}\right)^{-1} \int_{G_{j}}|u| d x, \\
& \Phi_{2}(u)=\max _{1 \leq j \leq J}\left(\operatorname{meas} G_{j}\right)^{-1} \int_{G_{j}}|\operatorname{grad} w|^{2} d x,
\end{aligned}
$$

where $G_{j}$ are some a priori chosen (small) subdomains,

$$
\Phi_{3}(e, u)=\int_{\Omega}\left(H \operatorname{grad} u \cdot \operatorname{grad} \varphi+\left(\mathcal{Z}^{0}[u]^{-}-p+2 \omega H\right) \varphi\right) d x,
$$

where

$$
\begin{aligned}
& \varphi \in X_{i}(\Omega)=\left\{\varphi \in H_{0}^{1}(\Omega): \varphi=1 \text { a.e. on } \Omega_{i}^{*}\right. \text { and } \\
& \left.\qquad \varphi=0 \text { a.e. on } \Omega_{k}^{*} \text { for all } k \neq i\right\} .
\end{aligned}
$$

REMARK 4.1. Arguing as in the proof of Lemma 1.1 and in Remark 1 we can deduce for $u(e) \in H^{2}(\Omega)$, on the basis of (1.12), that the functional $\Phi_{3}$ represents the resultant of the transverse reactive forces of the obstacle $\Omega_{i}^{*}$. In fact, we derive that

$$
\Phi_{3}(e, u(e))=\int_{\Omega} N_{z}(e, u(e)) \varphi d x=\int_{\Omega_{i}^{*}} N_{z}(e, u(e)) d x .
$$

Proposition 4.1. Let $e_{n} \in U_{\mathrm{ad}}, e_{n} \rightarrow e$ in $U$. Then $u\left(e_{n}\right) \rightarrow u(e)$ in $V$.

Proof. This is based on Theorem 2.1. Since $H$ is a fixed function, the verification of $(2.1)(\mathrm{i}) \&(\mathrm{ii})$ is obvious. Now we define

$$
\langle f, v\rangle=-2 \omega\langle H, v\rangle_{0} \quad \text { and } \quad\langle B e, v\rangle=\langle p, v\rangle_{0},
$$

so that $B: U \rightarrow V^{*}$ is a continuous mapping.

Lemma 4.1. Let $e_{n} \in U_{\mathrm{ad}}, e_{n} \rightarrow e$ in $U$. Then

$$
\Phi_{i}\left(e_{n}, u\left(e_{n}\right)\right) \rightarrow \Phi_{i}(e, u(e)), \quad i=1,2,3,
$$

as $n \rightarrow \infty$. 
Proof. Set $u_{n} \equiv u\left(e_{n}\right), u \equiv u(e)$. By Proposition 4.1, $u_{n} \rightarrow u$ in $V$. Then we may write

$$
\left|\int_{G_{j}}\left(\left|u_{n}\right|-|u|\right) d x\right| \leq \int_{G_{j}}\left|u_{n}-u\right| d x \leq\left(\operatorname{meas} G_{j}\right)^{1 / 2}\left\|u_{n}-u\right\|_{0} \rightarrow 0
$$

and

$$
\begin{aligned}
\left.\left.\left|\int_{G_{j}}\right| \operatorname{grad} u_{n}\right|^{2}-|\operatorname{grad} u|^{2}\right) & d x \mid \\
& \leq \int_{G_{j}}\left|\operatorname{grad}\left(u_{n}-u\right)\right|\left(\left|\operatorname{grad} u_{n}\right|+|\operatorname{grad} u|\right) d x \\
& \leq\left\|u_{n}-u\right\|_{1}\left(\left\|u_{n}\right\|_{1}+\|u\|_{1}\right) \rightarrow 0 .
\end{aligned}
$$

As a consequence, (4.2) follows for $i=1,2$.

Next, we have

$$
\begin{aligned}
\left|\Phi_{3}\left(e_{n}, u_{n}\right)-\Phi_{3}(e, u)\right|= & \mid \int_{\Omega}\left(H \operatorname{grad}\left(u_{n}-u\right) \cdot \operatorname{grad} \varphi\right. \\
& \left.+\left(\mathcal{Z}_{n}^{0}\left[u_{n}\right]^{-}-\mathcal{Z}^{0}[u]^{-}\right) \varphi+\left(p-p_{n}\right) \varphi\right) d x \mid \\
\leq & C\left(\left\|u_{n}-u\right\|_{1}+\left\|p-p_{n}\right\|_{\infty}\right. \\
& \left.+\int_{\Omega}\left(\left|\mathcal{Z}_{n}^{0}-\mathcal{Z}^{0}\right|\left[u_{n}\right]^{-}+\mathcal{Z}_{\max }\left|\left[u_{n}\right]^{-}-[u]^{-}\right|\right) d x\right) \\
\leq & \widetilde{C}\left(\left\|u_{n}-u\right\|_{1}+\left\|p-p_{n}\right\|_{\infty}+\left\|\mathcal{Z}_{n}-\mathcal{Z}\right\|_{\infty}\right) \rightarrow 0
\end{aligned}
$$

arguing as in the proof of (2.24) and (2.25).

TheOREM 4.1. The maximization problem (4.1) with any of the functionals $\Phi_{i}, i=1,2,3$, has at least one solution.

Proof. The functional

$$
J(e) \equiv \Phi_{i}(e, u(e)), \quad i=1,2,3,
$$

is continuous on the set $U_{\text {ad }}$ by Lemma 4.1. Since the set $U_{\text {ad }}$ is compact in $U$, there exists a maximizer $e^{*}$ in $U_{\mathrm{ad}}$.

Acknowledgements. The first author thankfully acknowledges the support of the Grant Agency of the Czech Republic under the grant 201/98/0528 and of the Ministry of Education, Youth and Sports under the grant OK-407.

\section{References}

[1] J. L. Armand, Application of the Theory of Optimal Control of Distributed Parameter Systems to Structural Optimization, NASA, 1972. 
[2] Y. Ben Haim and I. E. Elishakoff, Convex Models of Uncertainty in Applied Mechanics, Studies in Appl. Mech., 25, Elsevier, 1990.

[3] I. Hlaváček, Reliable solutions of elliptic boundary value problems with respect to uncertain data, Nonlinear Anal. 30 (1997), 3879-3890.

[4] I. Hlaváček and J. Lovíšek, Optimal design of an elastic plate with unilateral elastic foundation and rigid supports, using the Reissner-Mindlin plate model, Z. Angew. Math. Mech. 77 (1997), 377-385.

[5] J.-L. Lions, Quelques méthodes de résolution des problèmes aux limites non linéaires, Dunod, 1969.

[6] U. Mosco, Convergence of convex sets and solutions of variational inequalities, in: Constructive Aspects of Functional Analysis, Vol. II, G. Geymonat (ed.), Centro Intern. Matem., Estivo, 1971, 497-682.

[7] J. F. Rodrigues, Obstacle Problem in Mathematical Physics, North-Holland, 1987.

[8] L. Schwartz, Théorie des Distributions, Hermann, 1966.

Mathematical Institute

Academy of Sciences of the Czech Republic

Žitná 25

CZ-115 67 Praha 1, Czech Republic

E-mail: chleb@math.cas.cz

and

Institute of Computer Science

Pod vodárenskou věží 2

CZ-182 07 Praha 8, Czech Republic
Faculty of Civil Engineering

Slovak Technical University Radlinského 11

SK-813 68 Bratislava, Slovak Republic E-mail: bock@kmat.elf.stuba.sk 